\title{
HAMILTONIAN PATHS ON PLATONIC GRAPHS
}

\author{
BRIAN HOPKINS
}

Received 13 July 2003

\begin{abstract}
We develop a combinatorial method to show that the dodecahedron graph has, up to rotation and reflection, a unique Hamiltonian cycle. Platonic graphs with this property are called topologically uniquely Hamiltonian. The same method is used to demonstrate topologically distinct Hamiltonian cycles on the icosahedron graph and to show that a regular graph embeddable on the 2-holed torus is topologically uniquely Hamiltonian.
\end{abstract}

2000 Mathematics Subject Classification: 05C45, 05C62, 05C30.

1. Introduction. The five Platonic solids give rise to regular planar graphs with the additional property that each face is bordered by the same number of edges (including the "back face"). We use Schlegel diagrams to represent these graphs. A graph with exactly one Hamiltonian cycle is called uniquely Hamiltonian. The highly symmetric Platonic graphs admit many Hamiltonian cycles, but in some cases these cycles are very similar. Call a Platonic graph topologically uniquely Hamiltonian if all Hamiltonian cycles are equivalent under rotation and reflection. It is well known that the dodecahedron graph is topologically uniquely Hamiltonian. We develop a combinatorial method to establish this result and address related problems.

2. Platonic graphs. Given a Hamiltonian cycle on a Platonic graph, label each face with the number of its bordering edges that are used in the cycle. For the dodecahedral graph, a face label $F$ clearly satisfies $0 \leq F \leq 5$. In fact, it is easy to see that $3 \leq F \leq 4$. Since the dodecahedron graph is 3-regular, exactly two of the three edges incident with each vertex will be used in a Hamiltonian cycle. If $F \leq 2$, then at least one of the face's vertices will not be visited by the cycle, a contradiction. If $F=5$, then the cycle does not include any of the graph's other vertices (see Figure 2.1).

Consider an arbitrary Hamiltonian cycle on the dodecahedron graph. Let $x$ be the number of faces labeled 3 and $y$ the number of faces labeled 4 . Since there are twelve faces, $x+y=12$. The Hamiltonian cycle includes all twenty vertices and therefore consists of twenty edges. Since each edge borders two faces, the sum of the face labels is 40 , so $3 x+4 y=40$. The solution to this system of equations is $x=8$ and $y=4$. That is, any Hamiltonian cycle on the dodecahedron graph gives rise to eight faces labeled 3 and four faces labeled 4.

At least two of the faces labeled 4 must be adjacent. To show this, we attempt to find four mutually nonadjacent faces on the dodecahedron graph. Without loss of generality, suppose the middle face of the Schlegel drawing is labeled 4 (see Figure 2.2). Then the five faces adjacent to it cannot be chosen. There are two inequivalent choices 


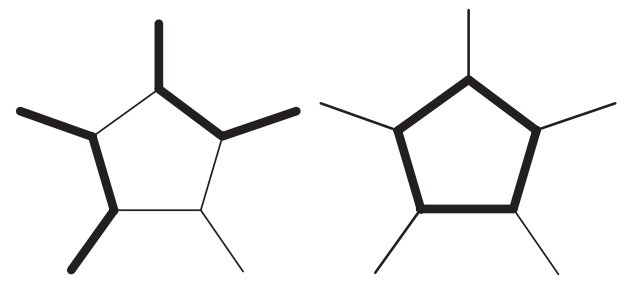

FIGURE 2.1

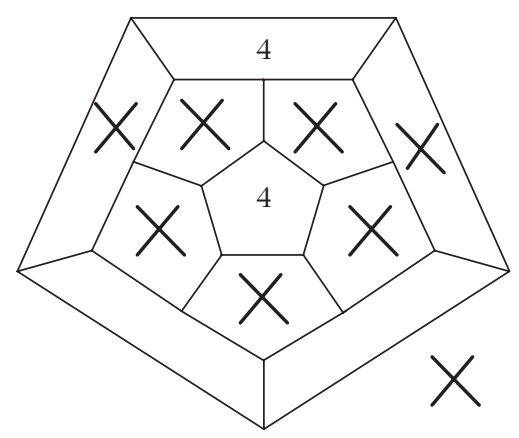

FIGURE 2.2

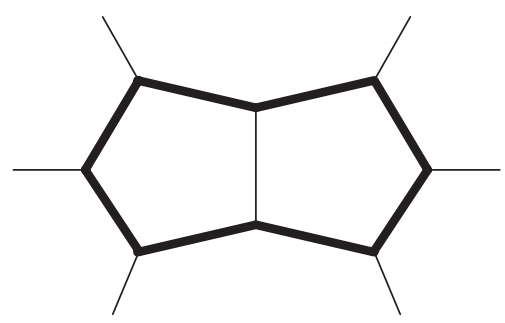

FIGURE 2.3

for the second face labeled 4. Choosing the "outside" face eliminates all other possibilities, a contradiction. Choosing any of the other faces leaves two adjacent faces for the remaining faces labeled 4 .

The edge shared by the two adjacent faces labeled 4 must be in any Hamiltonian cycle, else the cycle does not include half of the graph's vertices (see Figure 2.3). Since two of the three edges incident to each vertex are part of any Hamiltonian cycle, the unused edges for these two faces labeled 4 must be incident to the shared edge. The two remaining choices correspond to reflection being in the definition of topologically uniquely Hamiltonian.

This is enough to prove that the dodecahedron graph is topologically uniquely Hamiltonian. Without loss of generality, suppose the middle face in the Schlegel diagram and the face below it are labeled 4 , and choose one of the two options for the unused edges 


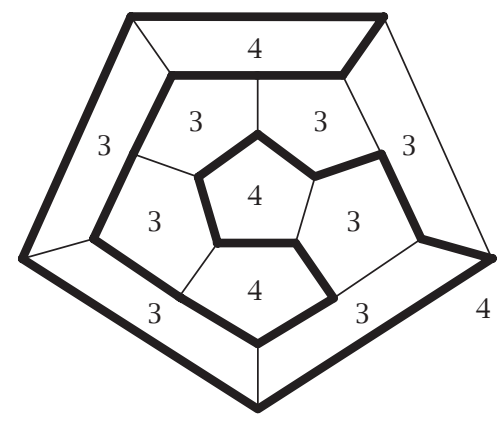

FIGURE 2.4
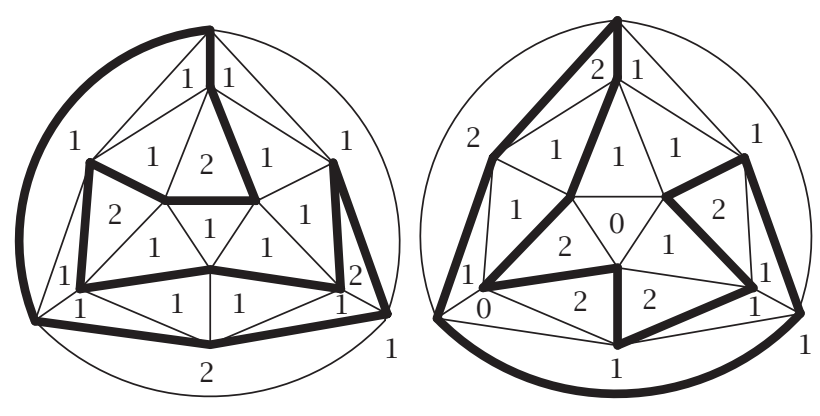

FIGURE 2.5

of these faces. The fact that two of the three edges incident to each vertex are part of any Hamiltonian cycle forces the rest of the cycle (see Figure 2.4). Notice that the faces labeled 4 are antipodal (on the original polyhedron).

This same technique shows that there are topologically distinct Hamiltonian cycles on the icosahedron graph; there are cycles that use at least one edge from every face, and cycles that do not (see Figure 2.5). It is easy to see that the tetrahedron and cube graphs are topologically uniquely Hamiltonian, while the octahedron graph is not.

3. Positive genus. Fortunately, this method is not limited to the five graphs corresponding to Platonic solids. In order for the idea of faces to be sensible, a graph must be embeddable on a 2-manifold. A $g$-Platonic graph is a regular graph that can be embedded on a genus $g$ torus such that each face is bordered by the same number of edges $[1,2]$. We can no longer work with Schlegel diagrams, but a $g$-torus can be represented as a $4 g$-gon with identified edges.

Consider the 2-Platonic graph with 16 vertices, 24 edges, and 6 faces, each an octagon. For a Hamiltonian path on this graph, clearly $4 \leq F \leq 7$. It is actually easy to show that $F=5$ and $F=7$ lead to contradictions. Following the reasoning above, the system of equations $x+y=6$ and $4 x+6 y=32$ has solutions $x=2$ and $y=4$. Again, using the fact that two of the three edges incident to each vertex are part of any Hamiltonian cycle forces the rest of the cycle (see Figure 3.1). 


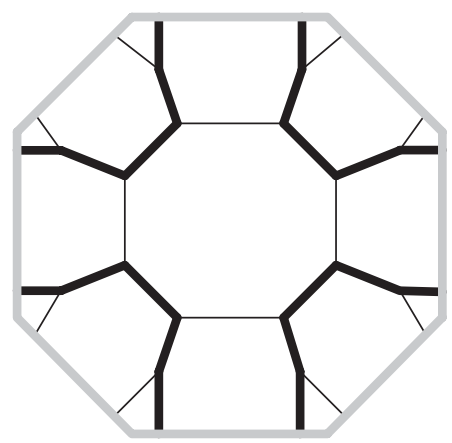

FIGURE 3.1

ACKNOWLEDGMENTS. Some of this material was developed in collaborative projects used at Seattle University, Saint Peter's College, Northwest Math Interaction, and the Northern New Jersey Professional Development \& Outreach Group of the Park City Mathematics Institute. Also, support for some of this research came from the Saint Peter's College Kenny Faculty Fellowship.

\section{REFERENCES}

[1] R. J. Trudeau, Dots and Lines, Kent State University Press, Ohio, 1976.

[2] _ Introduction to Graph Theory, Dover Publications, New York, 1993.

Brian Hopkins: Department of Mathematics, Saint Peter's College, Jersey City, NJ 07306, USA

E-mail address: bhopkins@spc.edu 


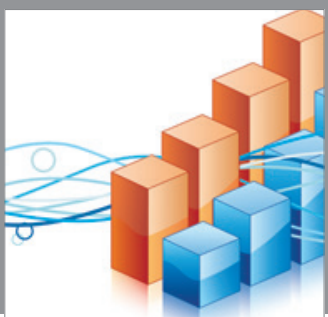

Advances in

Operations Research

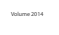

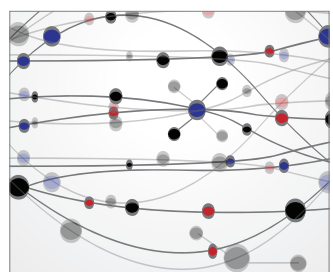

\section{The Scientific} World Journal
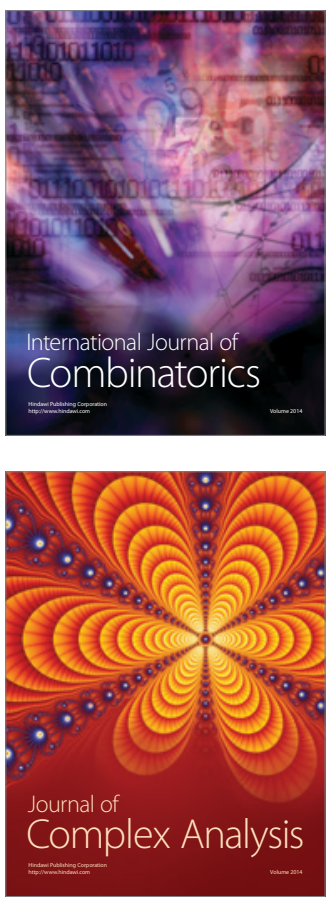

International Journal of

Mathematics and

Mathematical

Sciences
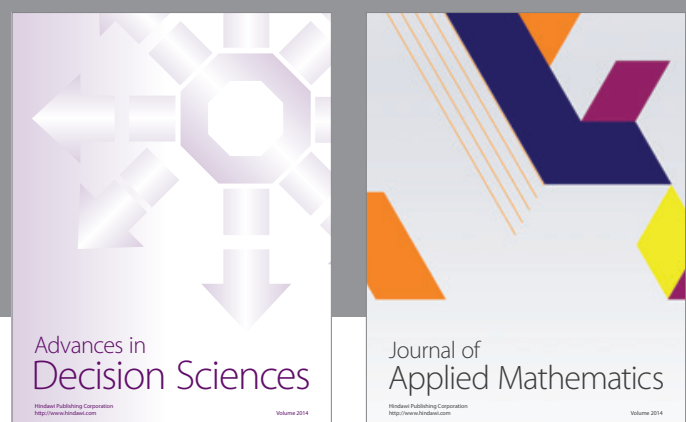

Journal of

Applied Mathematics
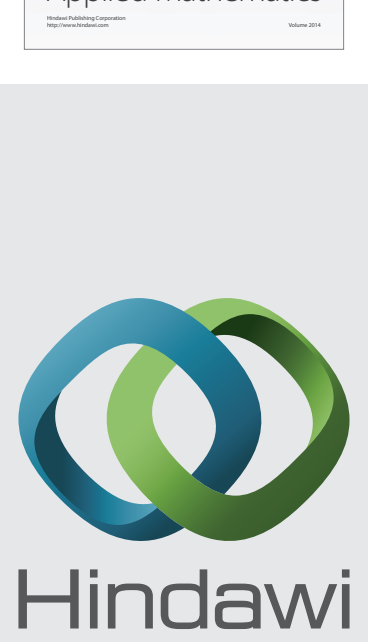

Submit your manuscripts at http://www.hindawi.com
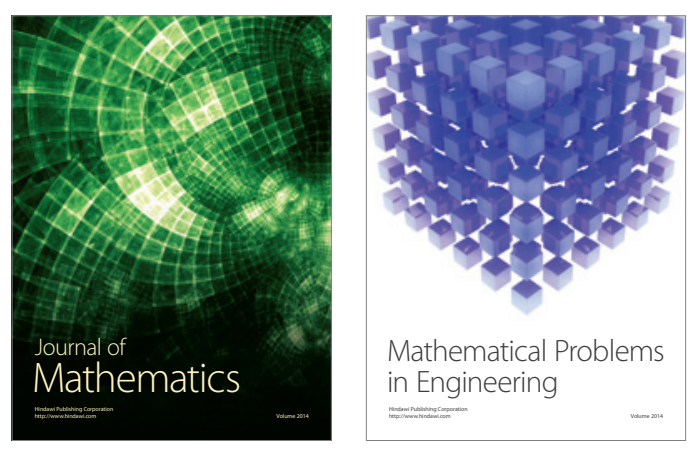

Mathematical Problems in Engineering
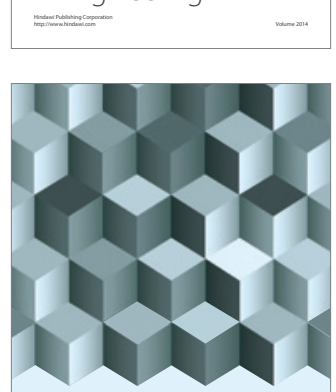

Journal of

Function Spaces
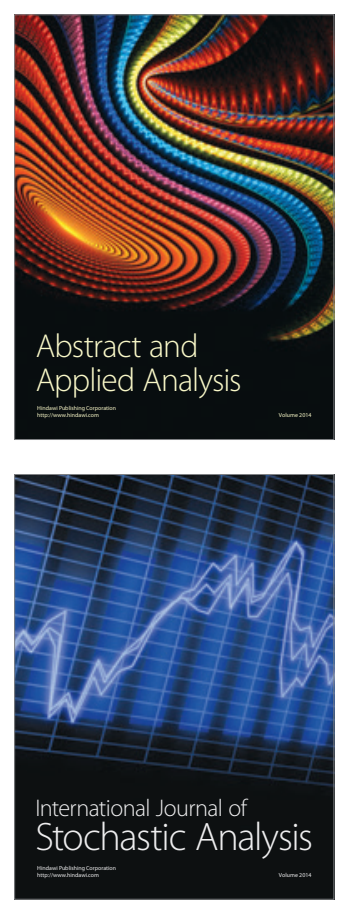

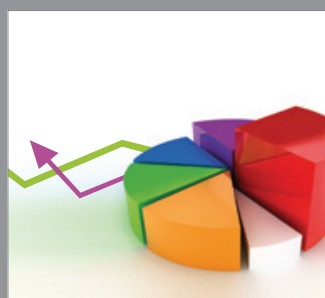

ournal of

Probability and Statistics

Promensencen
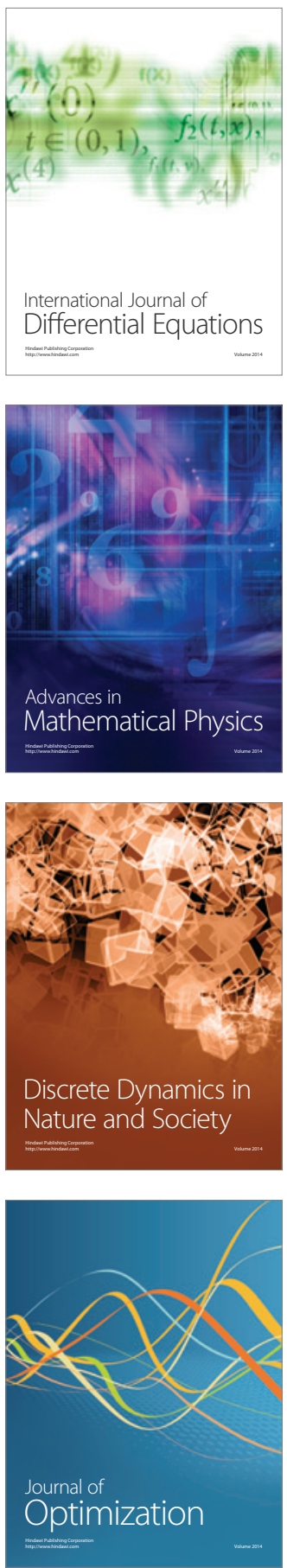